\title{
Recent Progress in Lunar Helium-3 Extraction Research
}

\author{
Aaron D.S. Olson \\ Engineering Physics Dept., University of Wisconsin-Madison ${ }^{1}$
}

\begin{abstract}
Research is ongoing to develop a prototype lunar volatiles extraction system that will demonstrate a process for acquiring helium-3 for future fusion power plants that would produce little to no radioactive waste, and other volatile gases that can be used for life support in space. The prototype system is called the Helium Extraction and Acquisition Test bed (HEAT). Testing of HEAT will focus on obtaining information on the rate of ${ }^{3} \mathrm{He}$ extraction possible and to what extent thermal energy recovery can be employed in this kind of volatile extraction system. Before demonstrating the evolution of ${ }^{3} \mathrm{He}$ out of regolith simulant, simulant that is embedded to a known concentration must be available in order to gauge the performance of HEAT. An implantation device is being developed to implant helium into batches of JSC-1A regolith simulant for HEAT. The implantation device under development is referred to as the Solar Wind Implanter (SWIM).
\end{abstract}

\section{Introduction and Background}

In 1986, researchers at the University of Wisconsin-Madison (UW-Madison) made the connection that helium-3 (a light isotope of helium) embedded in the lunar soil from the solar wind could be used to fuel nuclear fusion reactors that produce power without any radioactive waste (Wittenberg et al. 1986). Since then, research on the physics of producing power from two different helium-3 $\left({ }^{3} \mathrm{He}\right)$ fusion reactions has taken place along with research on the technology required to extract and collect ${ }^{3} \mathrm{He}$ from the loosely bound lunar top soil (or regolith) at the UWMadison Fusion Technology Institute (FTI). The FTI Inertial Electrostatic Confinement (IEC) fusion devices have been used to study the physics of ${ }^{3} \mathrm{He}$ fusion since the 1990's. Three designs of lunar ${ }^{3} \mathrm{He}$ miners have also been developed since 1988 at the FTI (Gajda 2006; Sviatoslavsky and Jacobs 1988; Sviatoslavsky 1993). Recently, a research effort on demonstrating the lunar ${ }^{3} \mathrm{He}$ extraction process outlined in the past miner designs, in a laboratory setting, has commenced (Olson 2013; Olson et al. 2015). The current progress of this effort will be further discussed in the following sections of this paper.

\section{Research Objectives}

The goal of this research is the development and testing of a prototype volatiles extraction system that will demonstrate a process for acquiring embedded ${ }^{3} \mathrm{He}$ from lunar regolith. This prototype system is referred to as the Helium Extraction and Acquisition Test bed (HEAT) and it is based on the past FTI Mark series miner designs. HEAT is currently being developed to process $157 \mathrm{~g} / \mathrm{s}$ of JSC-1A lunar regolith simulant, $1 / 1000^{\text {th }}$ of the mass flow rate of the Mark series designs. A conceptual model of the HEAT system is shown in Figure 1. The simulant to be processed will have a $20 \mathrm{ppb}$ concentration of implanted ${ }^{3} \mathrm{He}$. HEAT's thermal energy recovery efficiency and its rate of ${ }^{3} \mathrm{He}$ gas release will be evaluated relative to the Mark designs. Before demonstrating the evolution of helium out of regolith simulant, simulant that is embedded to a known concentration must be available in order to gauge the performance of the extraction system. Beyond the Apollo and Lunakhod lunar soil samples, there is no regolith or regolith

\footnotetext{
${ }^{1}$ The work presented in this paper would not have been possible without the support of the Wisconsin Space Grant Consortium Graduate Fellowship Award and the NASA Space Technology Research Fellowship Program
} 
simulant that has already been implanted with solar wind volatiles that is available for experimental studies. An implantation device is being developed to implant helium into batches of JSC-1A simulant. ${ }^{4} \mathrm{He}$ will be used at first and ${ }^{3} \mathrm{He}$ may be used after the device's operational performance is completely tested in order to keep costs down. The implantation device is referred to as the Solar Wind Implanter (SWIM).

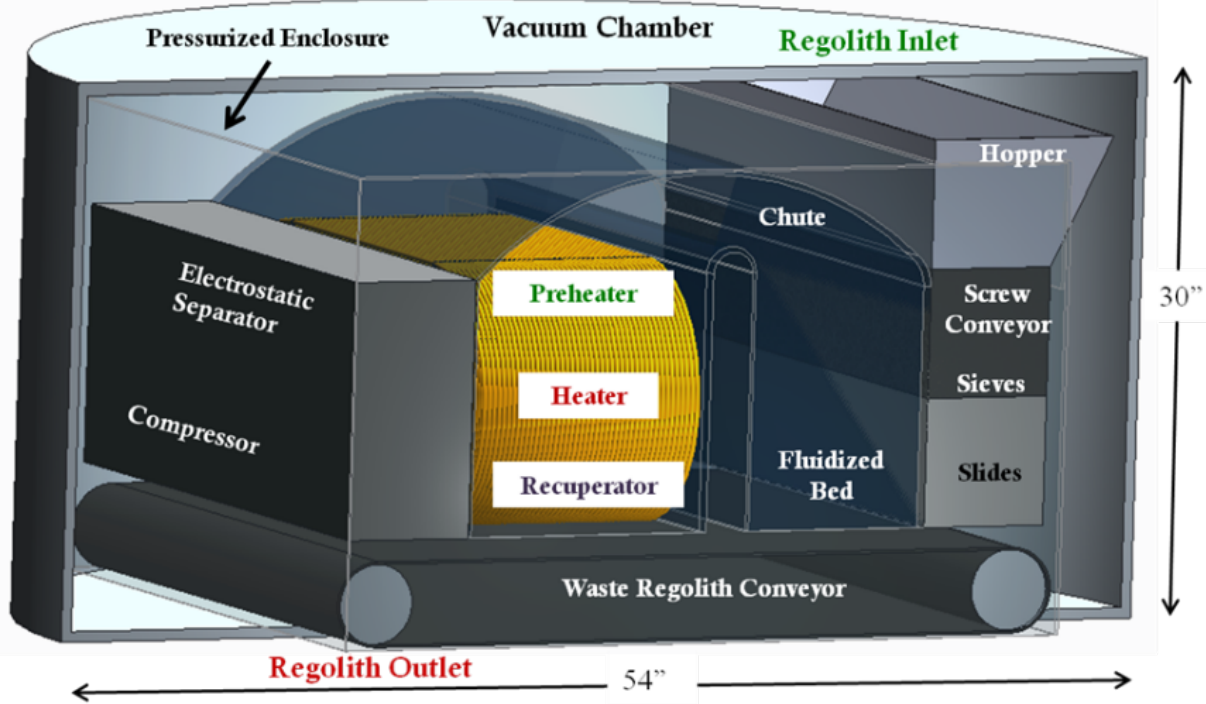

Figure 1. Concept of the HEAT system with a heat pipe heat exchanger shown in gold color

\section{Solar Wind Implanter Progress}

In the past, lunar analog materials such as the minerals Ilmenite and Olivine, have been implanted with helium using Plasma Source Ion Implantation (PSII) at UW-Madison (Kuhlman and Kulcinski 2012). The PSII method worked well enough for small samples of a few grams, but isn't practical for batches of hundreds of grams to a few kilograms of implanted simulant. The SWIM device is being developed to implant helium into batches of 5-100 micron JSC-1A regolith simulant. The concept for SWIM consists of having a controllable downward flow of simulant (coming out of a hopper/feeder device) that passes between planar electrode grids that produce a lateral (perpendicular to the falling simulant) flux of electrostatically accelerated helium ions inside of a vacuum chamber held below 100 mTorr of helium. The voltage potential between the electrodes determines the energy that the helium ions pick up before impacting the falling simulant particles. The solar wind that impacts the lunar surface is traveling between $300-900 \mathrm{~km} / \mathrm{s}$. To mimic that amount of energy $(\sim 1-2 \mathrm{keV} / \mathrm{amu})$, an electrostatic potential of around $4-8 \mathrm{kV}$ is required between a set of parallel electrodes. There are essentially four parts to SWIM: a hopper/feeder system, the system's support structure, the electrodes and tungsten filaments needed to drive helium ions into the simulant and a collection bin for the implanted simulant. These components can be seen in Figure 2. The process of implanting the JSC-1A simulant occurs in four steps. The first step is to fill the hopper/feeder with up to $2 \mathrm{~kg}$ of simulant before closing the vacuum chamber. Once a vacuum $(<10 \mathrm{mTorr})$ is pulled on the vacuum chamber, the system can be backfilled with helium to $\sim 100 \mathrm{mTorr}$. The third step is to turn on the high voltage power supply that provides the $8 \mathrm{kV}$ potential between the anode and cathode grids and to turn on the filament power supply that negatively biases and heats the tungsten filaments. The low pressure helium gas in the SWIM vacuum chamber is 
ionized via electron impact ionization, i.e., thermally released electrons from the tungsten filaments kick off electrons from helium atoms to produce helium ions. With the grid and filament power supplies on, a flux of helium ions will be accelerated through the cathode grids. It is currently estimated that with a $16 \mathrm{~V}$ potential across each filament will result in a current density through the grids that provides the $20 \mathrm{ppb}$ implantation desired. The fourth step is the controlled release of simulant from the hopper/feeder. A mass flow rate of $\sim 6 \mathrm{~g} / \mathrm{s}$ out of the feeder in a tenuous and thin stream allows for a sufficient dose into the simulant particles. Figure 3 illustrates the operation of the SWIM device and the placement of the grids relative to the stream of falling simulant.

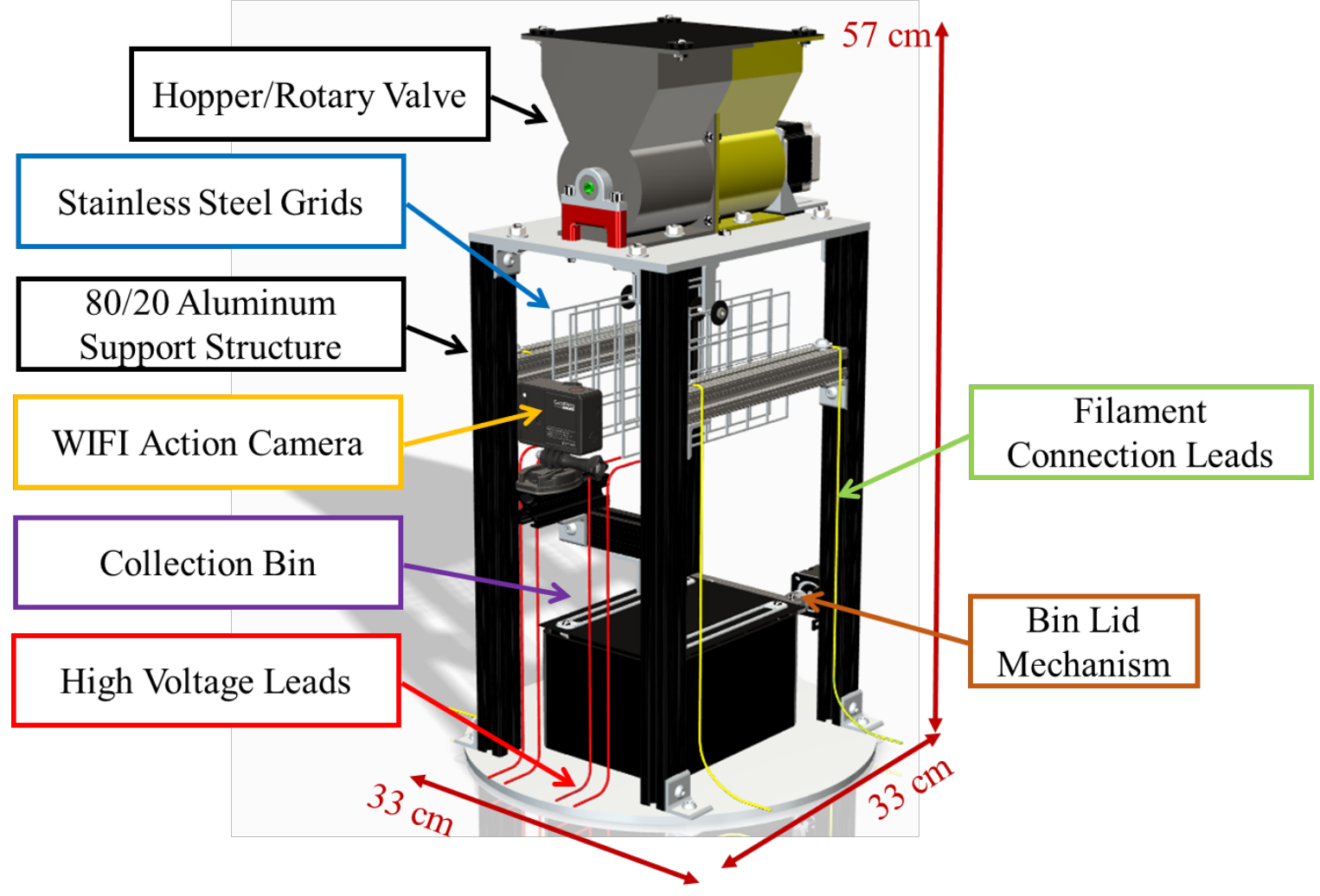

Figure 2. Components of the SWIM system 


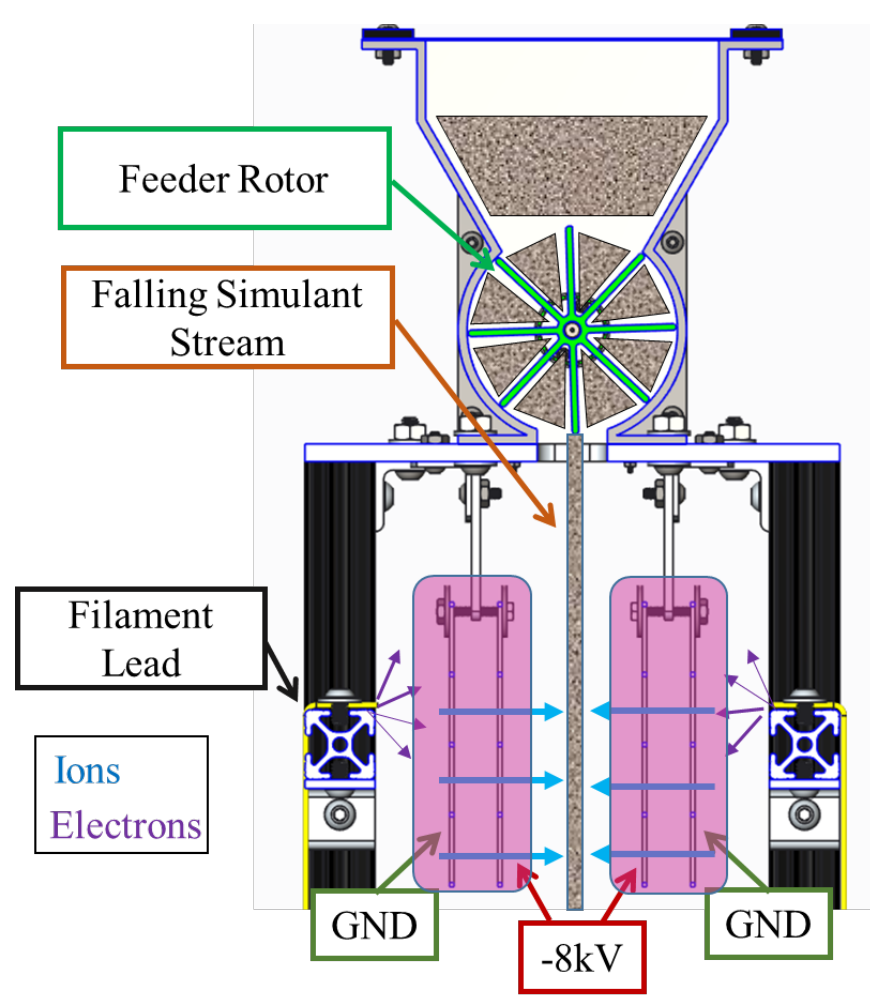

Figure 3. Operation of the SWIM device. The anode and cathode grids are $\sim 1.6 \mathrm{~cm}$ apart. Each grid is $\sim 18$ $\mathrm{cm}$ by $10 \mathrm{~cm}$ by $0.3 \mathrm{~cm}$ thick. Each grid is approximately $90 \%$ open with $2.5 \mathrm{~cm}$ width gap openings.

As of August of 2015, the first preliminary tests of the SWIM device have been completed. Figure 4 illustrates the operation of the SWIM device from multiple angles and during various operational steps. Simulant was dropped through a helium plasma discharge (where the ions flow), but the plasma current density and electrostatic potential were not adequate for the desired level of implantation in the initial tests. Design alterations/upgrades to the grid geometry are being worked on for future system operation. Furthermore, upgrades to the instrumentation system to measure the plasma current and a more reliable means to capture images and video of the simulant flow through the the plasma are also being developed.

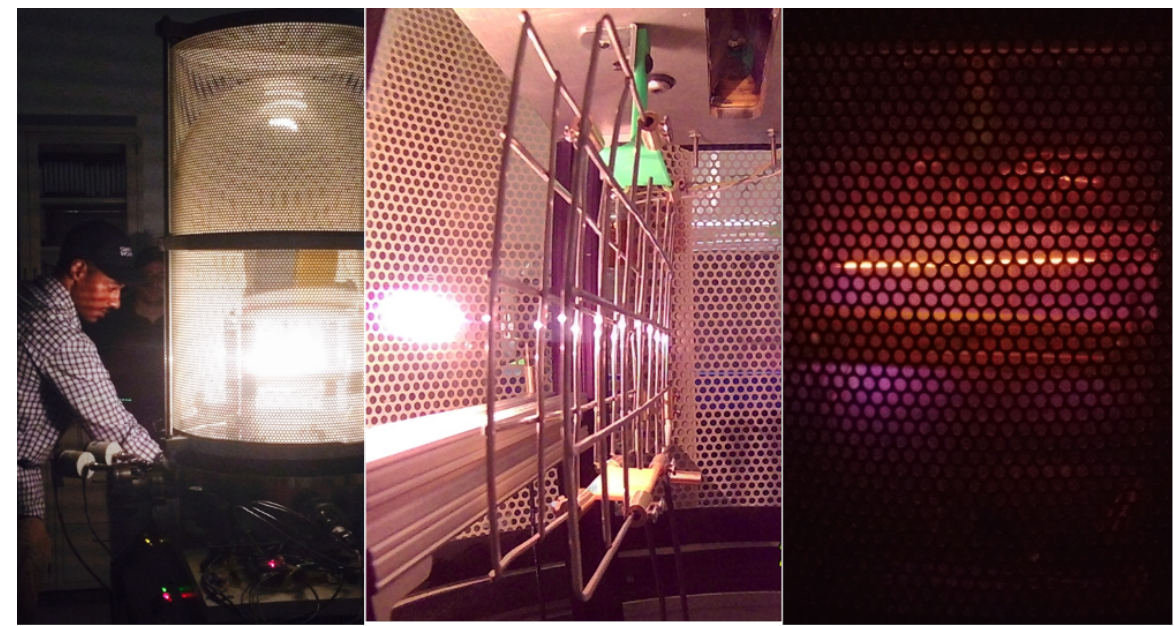

Filament on only
Action cam image
Filament on and discharge (side view)

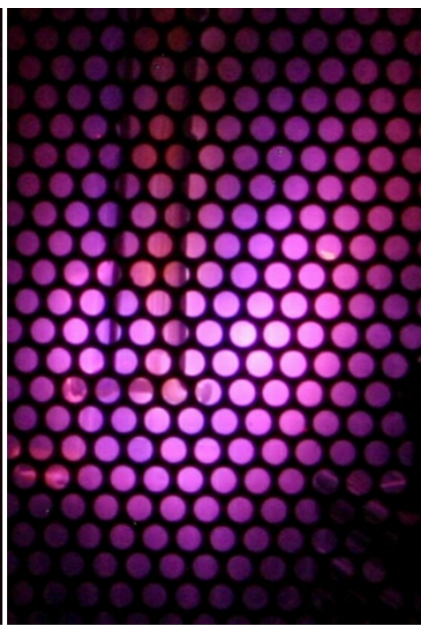

Discharge (front view)

Figure 4. Constructed SWIM system in various operation modes 


\section{Helium Extraction and Acquisition Test Bed Progress}

HEAT, when fully constructed, will heat simulant up to $700{ }^{\circ} \mathrm{C}$ in a moving bed heat pipe heat exchanger (HPHX). At this temperature $\sim 85 \%$ of the embedded helium will have diffused out of the simulant. At $\sim 1000{ }^{\circ} \mathrm{C}, \sim 100 \%$ of the embedded helium should be released, but the risk arises for the release of system damaging sulfur containing gases (Pepin et al. 1970). The use of heat pipes in the heat exchanger allows for the recuperation of thermal energy in the system, i.e., simulant that has already given up its implanted helium will pre-heat incoming volatile rich simulant. In the FTI lunar miner designs, a thermal energy recovery efficiency of $85 \%$ was selected. The heat pipes in HEAT are being designed to achieve this level of energy recovery. The previous lunar ${ }^{3} \mathrm{He}$ miner designs utilize concentrated solar thermal energy to heat the regolith. The HEAT system will use electrical resistance heating in place of solar thermal power. The heat transfer between the heat pipes and resistance heating elements and the regolith simulant within the HEAT device is dependent on the gravity driven velocity field of the simulant flowing through the device. Niegsch developed a model for granular flow through tube banks that takes into account the stagnation and void spaces that are characteristic of granular flow around obstacles. The model estimates a velocity field as well as the heat transfer coefficient between the tubes and the granular material (Niegsch et al. 1994). This model is being used to design the heat pipe arrangement for the HEAT system. Before constructing HEAT, testing of the simulant flow through a representative heat pipe arrangement of copper and stainless steel tubes is being done to validate the Niegsch model results with experimental data. This testing is occurring within a device referred to as the Granular Flow Experiment (GFX). The design of the GFX is illustrated in Figure 5 and a closer view of the pipe matrix is shown in Figure 6 along with the actual hardware. The GFX will be used to find the minimum inter-pipe spacing possible along with obtaining flow field and heat transfer experimental data. Particle image velocimetry (PIV) measurements from the GFX will be compared to the flow results of the Niegsch model and potentially granular CFD simulations as well. 


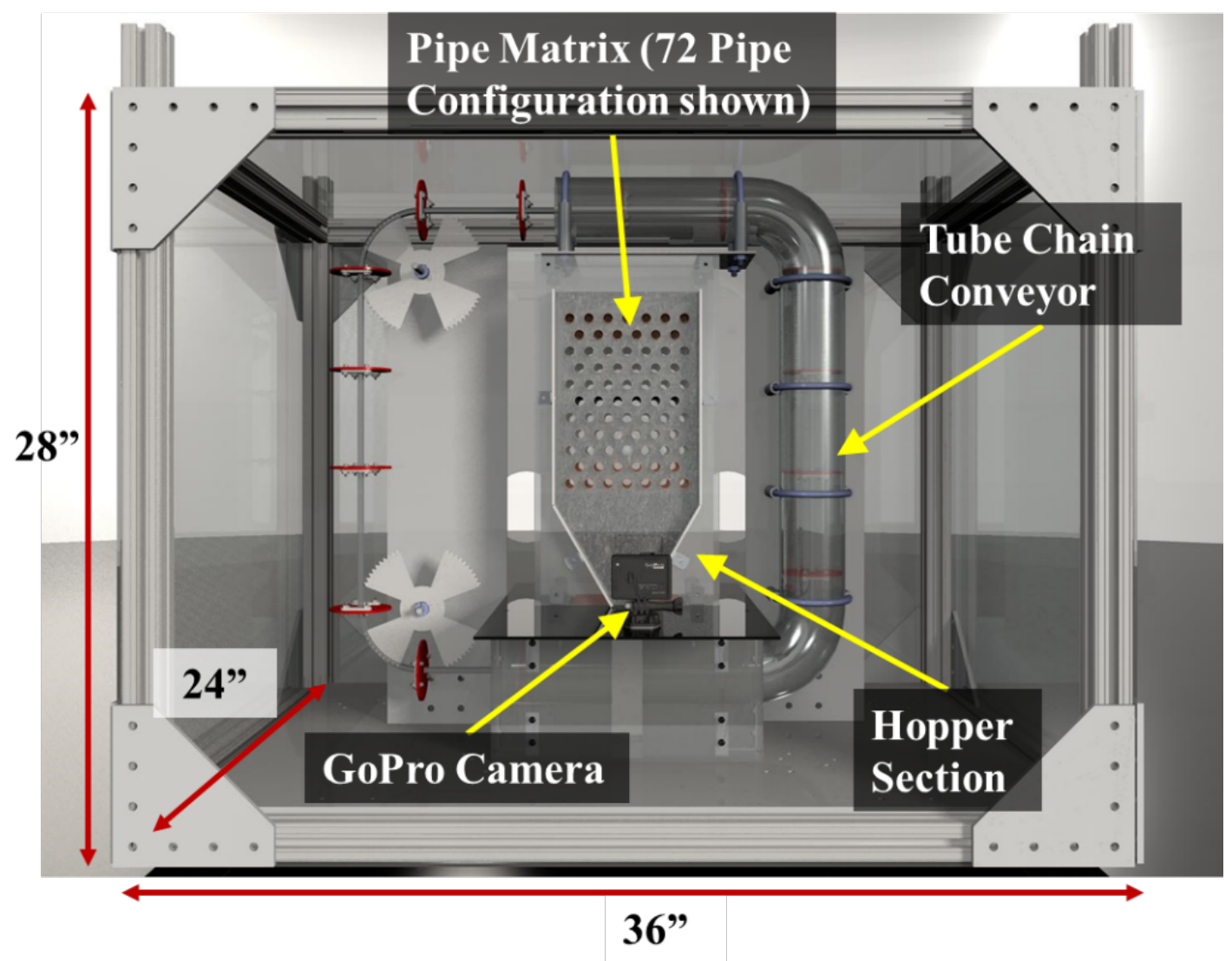

Figure 5. Updated GFX design illustrating the new pipe matrix/hopper design and tube chain conveyor.

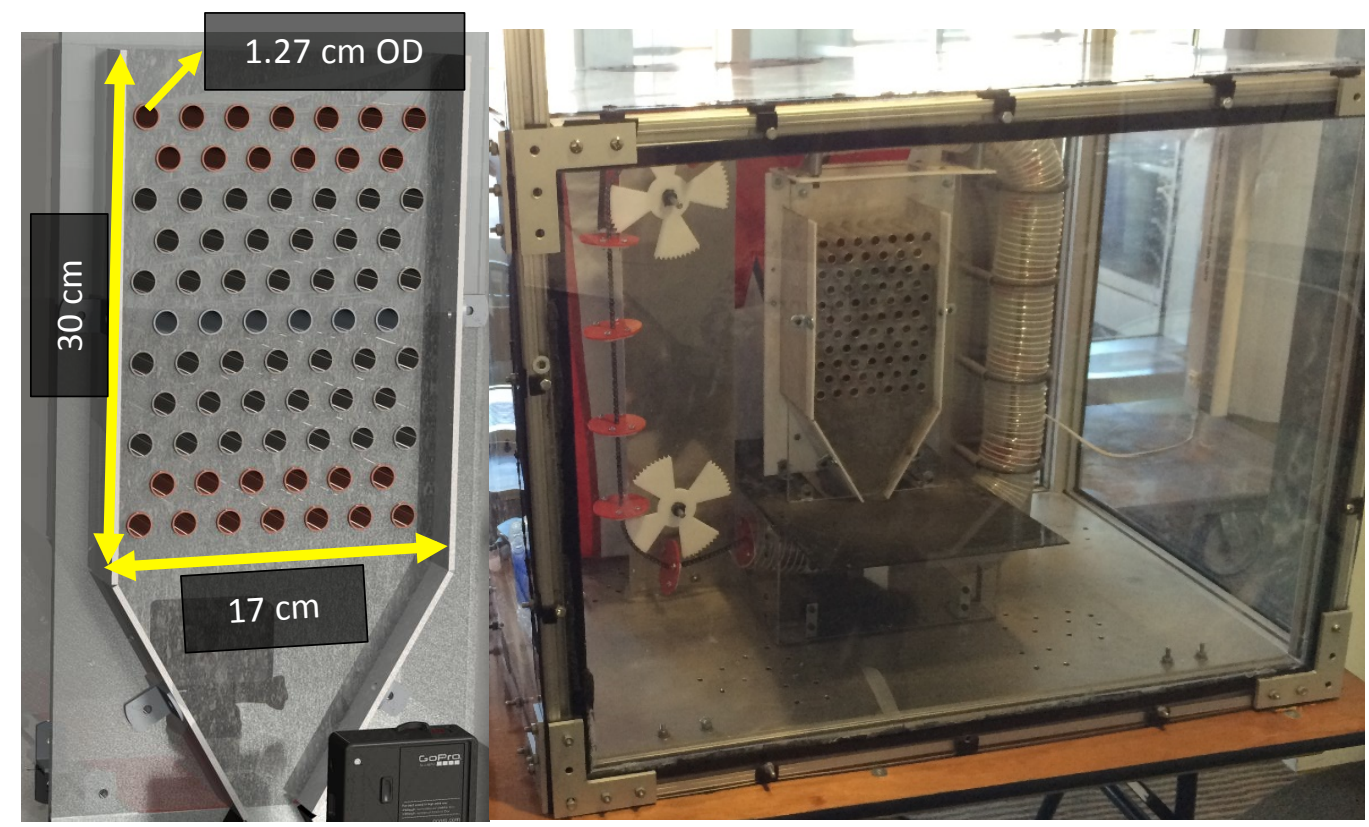

Figure 6. Pipe matrix cross section (left) and partially constructed GFX iteration 2 (right) at the 2015 Engineering Expo 


\section{Conclusion}

At the end of this research endeavor, a fully functional HEAT system will be constructed and tested for its continuous release rate of ${ }^{3} \mathrm{He}$ and its thermal energy recovery efficiency. The instrumentation system for HEAT will record temperature measurements and trace gas measurements. These measurements will be used to determine the thermal energy efficiency of the HEAT system. If the regolith reaches $700{ }^{\circ} \mathrm{C}$ in the center of the HEAT heating system and exits the heating system at $130{ }^{\circ} \mathrm{C}$, then the system will have an $85 \%$ thermal energy recovery efficiency. Trace gas concentration will be measured with an RGA. RGA measurements of the atmosphere inside of the HEAT system will give the concentration of helium as a function of time. The release rate of helium will be determined from these measurements. Based on the results of the functioning system, design improvements or alterations that will allow the system to achieve a higher release rate or a higher thermal energy recovery efficiency will be recommended. This will be key for future design work for a pilot scale lunar ${ }^{3} \mathrm{He}$ miner. A summary of the HEAT system's goals and the measurement criteria for these goals is shown in Table 1.

Table 1. Summary of HEAT Goals

Goal

${ }^{3} \mathrm{He}$ release rate of $17 \mathrm{mg} / \mathrm{hr}$ $\left(1 / 1000^{\text {th }}\right.$ of Mark series designs)

Achieve $85 \%$ thermal energy recovery efficiency

Recommendations to improve the system for a future lunar pilot scale miner

\section{Evaluation Criteria}

RGA measurements of atmosphere inside of the HEAT system

Change in temperature of the regolith simulant throughout the HEAT system as measured by thermocouples

Design alterations to increase the ${ }^{3} \mathrm{He}$ release rate and the energy recovery efficiency

\section{References}

Gajda, M. (2006). “A Lunar Volatiles Miner.” University of Wisconsin-Madison, M.S. Thesis.

Kuhlman, K., and Kulcinski, G. L. (2012). "Helium Isotopes in the Lunar Regolith - Measuring Helium Isotope Diffusivity in Lunar Analogs." Moon: Prospective Energy and Material Resources, Springer Praxis, 22-56.

Niegsch, J., Koneke, D., and Weinspach, P. M. (1994). "Heat transfer and flow of bulk solids in a moving bed." Chemical Engineering and Processing, 33, 73-89.

Olson, A. D. S. (2013). "The Mark IV: A Scalable Lunar Miner Prototype." International Astronautical Congress 2013, Beijing, China, IAC-13.A3.2B.7.

Olson, A. D. S., Santarius, J. F., and Kulcinski, G. L. (2015). "Design of a Lunar Solar Wind Volatiles Extraction System." Proceedings of the Wisconsin Space Conference, 1(1).

Pepin, R. O., Nyquist, L. E., Phinney, D., and Black, D. C. (1970). "Rare Gases in Apollo 11 Lunar Material." Proceedings of the Apollo 11 Lunar Science Conference, 1435 - 1454.

Sviatoslavsky, I. N. (1993). "The Challenge of Mining He-3 on the Lunar Surface: How All the Parts Fit Together." Space 94, The 4th International Conference and Exposition on Engineering, Construction and Operations in Space, and The Conference and Exposition/Demonstration on Robotics for Challenging Environments, February 26 - March 3, 1994, Albuquerque NM Also: WCS.

Sviatoslavsky, I. N., and Jacobs, M. K. (1988). "Mobile Helium-3 Mining and Extraction System and Its Benifits Toward Lunar Base Self-Sifficiency." Wisconsin Center for Space Automation and Robotics Technical Report (WCSAR-TR) AR3-8808-1.

Wittenberg, L. J., Santarius, J. F., and Kulcinski, G. L. (1986). "Lunar Source of He-3 for Commercial Fusion Power." Fusion Technology, 10, 167. 